\title{
Causes of hospitalization and predictors of HIV-associated mortality at the main referral hospital in Sierra Leone: a prospective study
}

Sulaiman Lakoh', Darlinda F. Jiba², Joseph E. Kanu', Eva Poveda ${ }^{3}$, Angel Salgado-Barreira ${ }^{4}$, Foday Sahr ${ }^{1}$, Momodu Sesay ${ }^{5}$, Gibrilla F. Deen ${ }^{1}$, Tom Sesay ${ }^{6}$, Wadzani Gashau², Robert A. Salata ${ }^{7,8}$ and George A. Yendewa ${ }^{7,8,9^{*}}$ (i)

\begin{abstract}
Background: HIV infection is a growing public health problem in Sierra Leone and the wider West Africa region. The countrywide HIV prevalence was estimated at 1.7\% (67,000 people), with less than 30\% receiving life-saving ART in 2016. Thus, HIV-infected patients tend to present to health facilities late, with high mortality risk.

Methods: We conducted a prospective study of HIV inpatients aged $\geq 15$ years at Connaught Hospital in Freetown —-the main referral hospital in Sierra Leone-from July through September 2017, to assess associated factors and predictors of HIV-related mortality.
\end{abstract}

Results: One hundred seventy-three HIV inpatients were included, accounting for 14.2\% (173/1221) of all hospital admissions during the study period. The majority were female (59.5\%, 70/173), median age was 34 years, with 51.4\% (89/173) of them diagnosed with HIV infection for the first time during the current hospitalization. The most common admitting diagnoses were anemia (48\%, 84/173), tuberculosis $(24.3 \%, 42 / 173)$, pneumonia $(17.3 \%, 30 / 173)$ and diarrheal illness $(15.0 \%, 26 / 173)$. CD4 count was obtained in $64.7 \%(112 / 173)$ of patients, with median value of

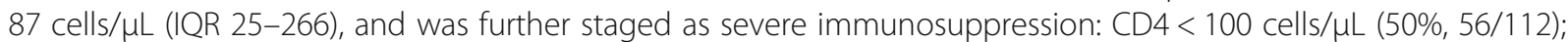

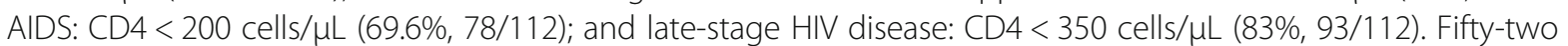
patients $(30.1 \%, 52 / 173)$ died during hospitalization, $23 \%(12 / 52)$ of them within the first week. The leading causes of death were anemia $(23.1 \%, 12 / 52)$, pneumonia $(19.2 \%, 10 / 52)$, diarrheal illness $(15.4 \%, 8 / 52)$ and tuberculosis $(13.6 \%, 7 / 52)$. Neurological symptoms, i.e., loss of consciousness $(p=0.04)$ and focal limb weakness $(p=0.04)$; alcohol use $(p=0.01)$; jaundice $(p=0.02)$; cerebral toxoplasmosis $(p=0.01)$; and tuberculosis $(p=0.04)$ were significantly associated with mortality; however, only jaundice (AOR $0.11,95 \% \mathrm{Cl}[0.02-0.65] ; p=0.01$ ) emerged as an independent predictor of mortality.

Conclusion: HIV-infected patients account for a substantial proportion of admissions at Connaught Hospital, with a high morbidity and in-hospital mortality burden. These findings necessitate the implementation of specific measures to enhance early HIV diagnosis and expand treatment access to all HIV-infected patients in Sierra Leone.

Keywords: HIV, Diagnosis, Mortality, In-hospital, Sierra Leone

\footnotetext{
*Correspondence: gay7@case.edu

${ }^{7}$ Department of Medicine, Case Western Reserve University School of Medicine, Cleveland, $\mathrm{OH}$, USA

${ }^{8}$ Division of Infectious Diseases and HIV Medicine, University Hospitals

Cleveland Medical Center, Cleveland, OH, USA

Full list of author information is available at the end of the article
}

(c) The Author(s). 2019 Open Access This article is distributed under the terms of the Creative Commons Attribution 4.0 International License (http://creativecommons.org/licenses/by/4.0/), which permits unrestricted use, distribution, and reproduction in any medium, provided you give appropriate credit to the original author(s) and the source, provide a link to the Creative Commons license, and indicate if changes were made. The Creative Commons Public Domain Dedication waiver (http://creativecommons.org/publicdomain/zero/1.0/) applies to the data made available in this article, unless otherwise stated. 


\section{Background}

Despite the global roll-out of antiretroviral therapy (ART), high in-hospital mortality associated with human immunodeficiency virus (HIV) infection remains a major problem in HIV care in resource-limited settings. The United Nations Joint Programme on HIV and AIDS (UNAIDS) 2017 Report has estimated that since the beginning of the global HIV pandemic in 1981, 35.4 million acquired immunodeficiency syndrome (AIDS)-related deaths have occurred worldwide-with 940,000 deaths recorded in 2017 alone despite death rates actually declining since 2005 [1]. A disproportionately high number of the global total deaths (70\%) occurred in sub-Saharan Africa, primarily in the high burden countries where the majority of HIV-infected people reside [1]. Of note, the proportion of deaths reported from the World Health Organization (WHO) region of West and Central Africa was particularly striking. Although countries in this WHO region have consistently reported low HIV seroprevalence rates for many years (generally < $4 \%$ ) [2], West and Central Africa accounted for 30\% (280, 000) of the global mortality in 2017 [1], highlighting a regional public health problem of growing magnitude and importance.

Several studies from West Africa have previously assessed the prevalence and associated factors of AIDS-related in-hospital mortality. A review of 207 HIV-infected patients admitted at a northern Nigerian tertiary health center recorded a $31.9 \%$ mortality rate (66 deaths) in 2012 [3]. Similarly, another study from Ghana $(n=547)$ reported an AIDS-associated mortality rate of $40.6 \%(222 / 547)$ during 2012-2013, with $55.9 \%(124 / 222)$ of those deaths occurring in the first week of hospitalization [4]. The most comprehensive study to date was conducted by the West Africa International epidemiological Database to Evaluate AIDS (IeDEA) group in 2010 and it included a total of 823 participants at clinical sites in 5 West African countries (Benin, Burkina Faso, Cote d'Ivoire, Mali and Senegal). This study recorded an in-hospital mortality rate of $38 \%$ [5]; however, given that it was conducted almost a decade ago before HIV services were scaled-up, the findings may not be reflective of current trends-hence, the need for an update from countries in the region.

In Sierra Leone, the HIV epidemic has steadily expanded ever since the first 10 cases were reported among commercial sex workers in 1987-and has been fueled, in part, by a severely under-resourced healthcare system, a decade long civil war (1991-2000) and disruptions in HIV care due to the recent Ebola epidemic (2014-2016) [6]. The latest countrywide HIV seroprelavance rate was estimated at $1.7 \%$ in 2016 , representing a total of $67,000 \mathrm{HIV}$-infected people, with less than $30 \%$ receiving ART [7]. Over a 6-year period (2010-2016), the country saw a $7 \%$ increase in new infections and a $6 \%$ rise in AIDS-related deaths [7]. In a cross-sectional study of newly diagnosed HIV-infected outpatients at the HIV clinic at Connaught Hospital in Freetown (the main referral hospital in Sierra Leone), we previously documented a high prevalence $(75 \%, n=155)$ of late stage HIV presentation among newly diagnosed patients, defined as CD $4<350$ and/or the presence of an AIDSdefining illness at diagnosis [8]. However, few studies have examined the disease progression patterns and clinical outcomes of HIV-infected patients in this West African country.

In this prospective study, we assessed the prevalence, clinical presentation, associated factors and predictors of in-hospital HIV-related mortality among adult HIVinfected patients admitted to Connaught Hospital in Freetown.

\section{Methods \\ Study population and setting}

A total of 173 HIV-infected patients aged $\geq 15$ years who were admitted at Connaught Hospital in Freetown were consecutively selected and included in the study from July through September 2017. Connaught Hospital is a 300-bed academic facility located in Freetown in Sierra Leone. It is also the main referral hospital for the management of medical and surgical cases in the country and is affiliated with the College of Medicine and Allied Health Sciences of the University of Sierra Leone, the country's only medical school. It has 10 wards (5 surgical, 4 medical, 1 pediatric), 1 intensive care unit, 2 accident and emergency wards (medical and surgical) and 2 private wards (for male and female patients, respectively). It is also the site of the largest HIV clinic and treatment center in the country. The facility offers both outpatient and in-patient services including HIV Counselling and Testing Services (HCTS), Prevention of Mother to Child Transmission (PMTCT) services, Antiretroviral Treatment (ART), and treatment of opportunistic infections.

\section{Clinical data collection and ethical considerations}

Demographic and clinical data were recorded. Informed written consent from participants aged $\geq 18$ years and written parental consent was obtained for patients aged 15-18 years prior to enrollment in the study. All patient personal information were deindentified before data were transferred into a password protected spreadsheet accessible only to study personnel. Ethical approval for the study was obtained from the Sierra Leone Ethics Scientific and Research Committee. 


\section{Diagnoses and definitions}

The fourth-generation rapid test by SD Bioline HIV-1/2 3.0 (Standard Diagnostics Inc) was used for HIV testing. CD4 cell count was determined using the Alere Pima ${ }^{\text {max }}$ Analyzer (Abbott), a point-of-care testing platform with comparable performance to flow cytometry-based methods and validated in resource-limited settings [9]. Tuberculosis was confirmed using the GeneXpert MTB/ RIF assay (Cepheid) and/or sputum acid fast bacilli (AFB) smear, with compatible chest radiographic findings. Cerebral toxoplasmosis was diagnosed clinically based on the presence of focal neurologic deficit(s) with/ without features of encephalitis and the presence of ring-enhancing cranial lesions on $\mathrm{CT}$ imaging. Other AIDS-defining illnesses (ADIs) such as cryptococcal meningitis, pneumocystis pneumonia, Kaposi sarcoma, and esophageal candidiasis were diagnosed on the basis of compatible clinical findings. Coinfections with malaria and hepatitis B virus (HBV) were confirmed with the appropriate serological tests in the setting of compatible physical and/or clinical findings. Sepsis was defined in accordance with the Third International Consensus Definitions for Sepsis, which considers a quick sequential organ failure assessment score (qSOFA score) $\geq 2$ in the setting of a suspected or confirmed microbiologic etiology as diagnostic of sepsis [10]. Anemia was defined as hemoglobin concentration of $<11.0 \mathrm{~g} / \mathrm{dL}$ for the adult non-pregnant population, which is the lower limit of the reference range at the Connaught Hospital Laboratory. Alcohol use was defined as consuming $>20 \mathrm{~g}$ or 2 drinks of an alcoholic beverage daily, while illicit drug use was defined as using any quantity of any of marijuana, cocaine or injected heroine in the last 30 days.

In all instances, assiduous attempts were made by the three physicians who collected the clinical data to identify and confirm the admitting diagnoses and causes of death on the basis of test results, diagnostic criteria and other available medical records. In the absence of these, compatible physical and/or clinical findings and other supporting clinical evidence were used to arrive at a consensus presumptive diagnosis or cause of death.

\section{Statistical analysis}

All statistical analyses were performed using the software package SPSS Statistics Version 25.0 (Armonk, NY: IBM Corp). Categorical variables were reported as frequency (percentage) and compared using the Pearson's chi-squared $\left(x^{2}\right)$ test. Continuous variables were recorded as median (interquartile range) and compared using the nonparametric independent samples MannWhitney U test or exact Fischer test, where appropriate. Associations were tested between in-hospital mortality and the following variables in the unadjusted univariate analysis: age, gender, specific risk factors (smoking, alcohol use, illicit drug use), CD4 count, WHO clinical staging, admitting diagnosis, ART status, and length of hospital stay in the unadjusted univariate analysis. A logistic regression model was used to determine the independent predictors of in-hospital mortality in the multivariate analysis. Other than age, gender and ART status which were a priori included, only variables that achieved $p<0.2$ in the univariate analysis were included in the multivariate regression analysis. To determine the odds of mortality with older age, age was further stratified into younger (15-44 years) and middle/older ( $\geq 45$ years) categories before inclusion in the model. In all analyses, $p<0.05$ was considered statistically significant.

\section{Results}

During the study period, a total of 1221 patients were admitted to the various wards at Connaught Hospital, representing an estimated bed occupancy rate of $95.0 \%$. About $32.9 \%$ of all hospitalized patients (402/1221) were admitted to the medical wards. Thus, the 173 HIVinfected individuals enrolled in our study represented $43.0 \%(173 / 402)$ of patients admitted to the medical wards and $14.2 \%(173 / 1221)$ of all hospital admissions during the study period.

\section{Baseline characteristics of study participants}

Table 1 presents the baseline characteristics of the study participants. Of a total of $173 \mathrm{HIV}$-infected patients who were included in the study, the majority were female $(59.5 \%, 103 / 173)$, with a median age of 34 years (IQR 28-44) and a median length of hospital stay of 12 days (IQR 8-17). Most participants had some form of education $(70.5 \%, 122 / 173)$, with the secondary level being the most frequently attained $(50.9 \%, 88 / 173)$. About twothirds reported being single. The proportion of patients who admitted to smoking, alcohol use, and illicit drug use were $20.2 \%$ (35/173), 22.5\% (39/173) and 5.2\% (9/ 173), respectively.

Over half of all study participants $(51.4 \%, 89 / 173)$ were diagnosed with HIV infection for the first time during the hospitalization; the remainder $(48.6 \%, 84 / 173)$ had a known history of HIV infection prior to hospitalization, with $25 \%(21 / 84)$ of them having been first diagnosed within the preceding 12 months. About 6\% (10/173) could not confirm whether they had been previously diagnosed. The majority were infected with HIV-1 type $(96.0 \%, 166 / 173)$, while $4 \%$ (7/173) of patients had HIV test results that were indeterminate for virus type. No cases of HIV-2 mono-infection or HIV-1/HIV-2 dual infection were detected.

In total, only $37.0 \%(64 / 173)$ of study participants were receiving ART during the hospital stay. The most common regimen was tenofovir/lamivudine/efavirenz (84.4\%, $54 / 64)$; the remaining patients were on zidovudine/ 
Table 1 Baseline characteristics of newly admitted HIV-patients at Connaught Hospital

\begin{tabular}{|c|c|}
\hline Characteristics & $\begin{array}{l}N(\%) \\
173(100 \%) \\
\end{array}$ \\
\hline \multicolumn{2}{|l|}{ Gender } \\
\hline Male & $70(40.5)$ \\
\hline Female & $103(59.5)$ \\
\hline \multicolumn{2}{|l|}{ Age, years } \\
\hline Median, IQR & $34(28-44)$ \\
\hline $15-24$ & $25(14.5)$ \\
\hline $25-34$ & $68(39.3)$ \\
\hline $35-44$ & $40(23.1)$ \\
\hline $45-54$ & $26(15.0)$ \\
\hline $55-64$ & $9(5.2)$ \\
\hline$>64$ & $5(2.9)$ \\
\hline \multicolumn{2}{|l|}{ Educational level } \\
\hline None & $51(29.5)$ \\
\hline Primary & $19(11.0)$ \\
\hline Secondary & $88(50.9)$ \\
\hline Tertiary & $15(8.7)$ \\
\hline \multicolumn{2}{|l|}{ Marital Status } \\
\hline Married & $52(30.1)$ \\
\hline Single & $116(67.0)$ \\
\hline Unknown & $5(2.9)$ \\
\hline \multicolumn{2}{|l|}{ Occupation } \\
\hline Trader/Business & $68(39.3)$ \\
\hline Student & $19(11.0)$ \\
\hline Unemployed & $4(2.3)$ \\
\hline Other & $82(47.4)$ \\
\hline \multicolumn{2}{|l|}{ Risk factors } \\
\hline Smoking & $35(20.2)$ \\
\hline Alcohol use & $39(22.5)$ \\
\hline Illicit drugs & $9(5.2)$ \\
\hline \multicolumn{2}{|l|}{ HIV status } \\
\hline \multicolumn{2}{|l|}{ Known before admission } \\
\hline$<12$ months & $21(12.2)$ \\
\hline$>12$ months & $53(30.6)$ \\
\hline Diagnosed during hospitalization & $89(51.4)$ \\
\hline Unknown & $10(5.8)$ \\
\hline \multicolumn{2}{|l|}{ ART regimens, $N=64$ (100\%) } \\
\hline $\mathrm{TDF}+3 \mathrm{TC}+\mathrm{EFV}$ & $54(84.4)$ \\
\hline$A Z T+3 T C+N V P$ & $7(10.9)$ \\
\hline $\mathrm{AZT}+3 \mathrm{TC}+\mathrm{EFV}$ & $1(1.6)$ \\
\hline$A Z T+3 T C+L P V / r$ & $2(3.1)$ \\
\hline \multicolumn{2}{|l|}{ CD4 count, cells/uL, N= 112 (100\%) } \\
\hline Median, IQR & $87(25-266)$ \\
\hline$<100$ & $56(32.3)$ \\
\hline
\end{tabular}

Table 1 Baseline characteristics of newly admitted HIV-patients at Connaught Hospital (Continued)

\begin{tabular}{ll}
\hline Characteristics & $N(\%)$ \\
& $173(100 \%)$ \\
\hline $100-199$ & $22(12.7)$ \\
$200-349$ & $15(8.7)$ \\
$\geq 350$ & $19(11.0)$ \\
Not obtained & $61(35.3)$ \\
WHO clinical staging & \\
Stage 1 & $1(0.6)$ \\
Stage 2 & $28(16.1)$ \\
Stage 3 & $115(66.5)$ \\
Stage 4 & $29(16.8)$ \\
\hline IQR interquartile range, AZT zidovudine, TDF tenofovir disoproxil fumarate, 3TC \\
lamivudine, EFV efavirenz, NVP nevirapine, LPV/r lopinavir/ritonavir
\end{tabular}

lamivudine/nevirapine (10.9\%, 7/64); zidovudine/lamivudine/lopinavir/ritonavir $(3.1 \%, 2 / 64)$; and zidovudine/ lamivudine/efavirenz $(1.6 \%, 1 / 64)$. Ninety-six percent (166/173) of all patients were receiving cotrimoxazole prophylaxis during hospital stay, in line with current WHO recommendations for treatment of HIV infection in resource-limited settings [11].

\section{Clinical presentation and admitting diagnoses}

Table 2 shows the clinical presentation (signs and symptoms) and admitting diagnoses of the study participants. The majority $(94.2 \%, 163 / 173)$ reported constitutional symptoms; other symptoms by systems included cardiopulmonary $(63.6 \%, 110 / 173)$, gastrointestinal $(54.9 \%, 95 /$ $173)$, and neurological $(37.0 \%, 64 / 173)$ and genitourinary $(15.0 \%, 26 / 173)$. The most common symptoms were fever $(77.5 \%, 163 / 173)$, cough $(56.1 \%, 110 / 173)$, weight loss $(53.8 \%, 134 / 173)$, generalized malaise $(53.2 \%$, 92/ $173)$, anorexia $(38.7 \%, 67 / 173)$, and diarrhea $(38.2 \%, 95 /$ 173).

The most common admitting diagnosis was anemia (48.0\%, 83/173 in total; in $15.0 \%(26 / 173)$ of cases as the primary admitting diagnosis and in 33\% (57/173) of cases associated with other admitting diagnosis), followed by tuberculosis $(24.3 \%, 42 / 173)$, pneumonia (17.3\%, 30/173), diarrheal illness (15.0\%, 26/173), malaria $(6.4 \%, 11 / 173)$, cerebral toxoplasmosis $(5.2 \%, 9 / 173)$, sepsis $(3.5 \%, 6 / 173)$, hepatitis B virus (HBV)-related decompensated liver cirrhosis $(2.9 \%, 5 / 173)$, gastroenteritis (2.9\%, 5/173), disseminated Kaposi sarcoma (2.3\%, 4/ $173)$, esophageal candidiasis $(2.3 \%, 4 / 173)$ and cryptococcal meningitis $(1.7 \%, 3 / 173)$.

\section{Immunological profile and WHO clinical staging}

The immunological profile of participants was assessed (Table 1). The CD4 count was obtained for only $64.7 \%$ $(112 / 173)$ of patients during the hospital stay. The 
Table 2 Presenting signs and symptoms and admitting diagnoses of HIV in-patients

\begin{tabular}{|c|c|}
\hline Presenting Signs, Symptoms and Diagnoses & $\begin{array}{l}N(\%) \\
173(100)\end{array}$ \\
\hline Constitutional & $163(94.2)$ \\
\hline Fever & $134(77.5)$ \\
\hline Weight loss & $93(53.8)$ \\
\hline Malaise & $92(53.2)$ \\
\hline Anorexia & $67(38.7)$ \\
\hline Night sweats & $20(11.6)$ \\
\hline Rash & $17(9.8)$ \\
\hline Neurological & $64(37.0)$ \\
\hline Headache & $33(19.1)$ \\
\hline Seizure & $2(1.2)$ \\
\hline Loss of consciousness & $23(13.3)$ \\
\hline Neck stiffness & $5(2.9)$ \\
\hline Limb weakness & $8(4.6)$ \\
\hline Cardiopulmonary & $110(63.6)$ \\
\hline Cough & $97(56.1)$ \\
\hline Chest pain & $39(22.5)$ \\
\hline Dyspnea & $29(16.8)$ \\
\hline Hemoptysis & $2(1.2)$ \\
\hline Gastrointestinal & $95(54.9)$ \\
\hline Diarrhea & $67(38.2)$ \\
\hline Abdominal pain & $34(19.7)$ \\
\hline Nausea/vomiting & $33(19.1)$ \\
\hline Jaundice & $7(4.0)$ \\
\hline Ascites & $9(5.2)$ \\
\hline Hepatosplenomegaly & $15(8.7)$ \\
\hline Odynophagia & $11(6.4)$ \\
\hline Genitourinary & $26(15.0)$ \\
\hline Frequency & $9(34.6)$ \\
\hline Dysuria & $4(2.3)$ \\
\hline Urgency & $3(1.7)$ \\
\hline Hematuria & $2(1.2)$ \\
\hline Genital ulcers & $3(1.7)$ \\
\hline \multicolumn{2}{|l|}{ Admitting diagnoses } \\
\hline Anemia & $83(48.0)$ \\
\hline Primary diagnosis & $26(15.0)$ \\
\hline In association with other conditions & $57(33.0)$ \\
\hline Tuberculosis & $42(24.3)$ \\
\hline Pneumonia & $30(17.3)$ \\
\hline Diarrheal illness & $26(15.0)$ \\
\hline Malaria & $11(6.4)$ \\
\hline Cerebral toxoplasmosis & $9(5.2)$ \\
\hline Sepsis & $6(3.5)$ \\
\hline Decompensated liver cirrhosis & $5(2.9)$ \\
\hline
\end{tabular}

Table 2 Presenting signs and symptoms and admitting diagnoses of HIV in-patients (Continued)

\begin{tabular}{ll}
\hline Presenting Signs, Symptoms and Diagnoses & $N(\%)$ \\
\hline Gastroenteritis & $173(100)$ \\
Disseminated Kaposi sarcoma & $5(2.9)$ \\
Esophageal candidiasis & $4(2.3)$ \\
Cryptococcal meningitis & $4(2.3)$ \\
Pneumocystis pneumonia & $3(1.7)$ \\
Drug-induced hepatotoxicity & $3(1.7)$ \\
Salmonella typhi infection & $1(0.6)$ \\
\hline
\end{tabular}

median CD4 count was 87 cells/ $\mu \mathrm{L}$ (IQR $25-266$ ). The distribution of patients based on CD4 count was further stratified as follows: CD $4<100$ cells $/ \mu \mathrm{L}$, i.e., severe immunosuppression $50 \%$ (56/112); CD $4<200$ cells $/ \mu \mathrm{L}$, i.e., AIDS $69.6 \%$ (78/112); and CD4 $<350$ cells $/ \mu \mathrm{L}$, i.e., latestage HIV disease $83.0 \%$ (93/112).

The WHO staging was obtained for all patients, with the majority $(83.2 \%, 114 / 173)$ being classified as either WHO stage 3 or 4 (Table 1 ).

\section{Distribution of AIDS-defining conditions}

The distribution of AIDS-defining conditions diagnosed (not mutually exclusive) was as follows: tuberculosis (24.3\%, 42/173), cerebral toxoplasmosis $(5.2 \%, 9 / 173)$, esophageal candidiasis $(2.3 \%, 4 / 173)$, Kaposi sarcoma $(2.3 \%, 4 / 173)$, Pneumocystis pneumonia $(1.7 \%, 3 / 173)$, cryptococcal meningitis $(1.7 \%, 3 / 173)$, and Salmonella typhi infection $(0.6 \%, 1 / 173)$ (Table 2).

\section{Causes of death}

A total of 52 deaths occurred during hospitalization, yielding a HIV-associated in-hospital mortality rate of $30.1 \%$. About $23.1 \%(12 / 52)$ of all deaths occurred within the first 7 days of hospitalization. The leading causes of death were anemia $(23.1 \%, 12 / 52)$, pneumonia $(19.2 \%$, $10 / 52)$, diarrheal illness $(15.4 \%, 8 / 52)$ and tuberculosis $(13.6 \%, 7 / 52)$. Table 3 displays the other causes of death stratified along ART-usage, given the low proportion (37\%) of patients who were on life-saving ART prior to hospitalization.

Associated factors and predictors of in-hospital mortality In the univariate analysis, alcohol use $(34.6 \%$ versus $14.4 \%, p=0.01)$; presence of jaundice $(9.6 \%$ versus $1.7 \%$, $p=0.02)$; presenting with limb weakness $(9.6 \%$ versus $2.5 \%, p=0.04)$; loss of consciousness $(9.6 \%$ versus $2.5 \%$, $p=0.04)$; tuberculosis $(13.5 \%$ versus $28.1 \%, p=0.04)$; or cerebral toxoplasmosis $(11.5 \%$ versus $2.5 \%, p=0.01)$ were significantly associated with in-hospital mortality (Table 4). No association was observed based on age, gender, CD4 count, WHO clinical stage, ART status or 
Table 3 Causes of death in hospitalized HIV-infected patients related to ART status

\begin{tabular}{|c|c|c|c|c|}
\hline \multirow[t]{2}{*}{ Cause of death } & \multirow{2}{*}{$\begin{array}{l}\text { Total } \\
N=52 \\
(100 \%)\end{array}$} & \multicolumn{2}{|l|}{ ART status } & \multirow[t]{2}{*}{$P$ value } \\
\hline & & $\begin{array}{l}\text { No } \\
N=36(100 \%)\end{array}$ & $\begin{array}{l}\text { Yes } \\
N=16(100 \%)\end{array}$ & \\
\hline Anemia & $12(23.1)$ & $10(27.8)$ & $2(12.5)$ & 0.17 \\
\hline Pneumonia & $10(19.2)$ & $7(19.4)$ & $3(18.8)$ & 0.95 \\
\hline Diarrheal illness & $8(15.4)$ & $3(8.3)$ & $5(31.3)$ & 0.04 \\
\hline Tuberculosis & $7(13.6)$ & $4(11.0)$ & $3(18.8)$ & 0.46 \\
\hline Cerebral toxoplasmosis & $6(11.5)$ & $5(13.9)$ & $1(6.2)$ & 0.43 \\
\hline Decompensated cirrhosis & $3(5.8)$ & $2(5.6)$ & $1(6.2)$ & 0.92 \\
\hline Pneumocystis pneumonia & $1(1.9)$ & $1(2.8)$ & - & 0.50 \\
\hline Malaria & $1(1.9)$ & $1(2.8)$ & - & 0.50 \\
\hline Bacterial meningitis & $1(1.9)$ & $1(2.8)$ & - & 0.50 \\
\hline Sepsis & $1(1.9)$ & - & $1(6.2)$ & 0.13 \\
\hline Drug-induced hepatotoxicity & $1(1.9)$ & $1(2.8)$ & - & 0.50 \\
\hline Stroke & $1(1.9)$ & $1(2.8)$ & - & 0.50 \\
\hline
\end{tabular}

length of hospital stay. In the multivariate logistic regression analysis, younger age (15-44 years), middle/older age ( $\geq 45$ years), gender, ART status and variables with $p<0.2$ in the univariate analysis were included. After adjusting the model, jaundice (AOR 0.11, 95\% CI [0.020.62]; $p=0.01$ ) was identified as the only independent predictor of in-hospital mortality (Table 5).

\section{Discussions}

This is the first study to assess HIV-related admissions and in-hospital mortality at the main referral health center in Sierra Leone. A high prevalence of HIV-associated in-hospital mortality $(30.1 \%, 52 / 173)$ was observed in a cohort of $173 \mathrm{HIV}$-infected individuals who were admitted to the medical wards at Connaught Hospital in Freetown during July to September in 2017. About 22\% $(12 / 52)$ of those deaths occurred within the first week of hospitalization. The median length of hospital stay was 12 days. The in-hospital mortality rate was similar to that obtained from the northern Nigerian cohort discussed earlier [3] but lower than findings from Ghana [4] and the 2010 West Africa IeDEA cohort study [5]. This likely reflects the higher burden of HIV disease and/or local healthcare challenges in the clinical management of patients in those countries before HIV services and ART programs were scaled-up. Furthermore, over $50 \%(89 / 173)$ of patients were diagnosed with HIV infection for the first time during hospitalization versus $24 \%$ in the West Africa IeDEA study [5] - not a surprising finding given that even though there are high levels of HIV awareness among adults in Sierra Leone (over 94\%), uptake in voluntary HIV testing has remained below $30 \%$ in the country [12].

Tuberculosis was one of the leading admitting diagnoses $(24.3 \%, 42 / 173)$ and although it was not the leading cause of death, it accounted for a substantial proportion of deaths $(13.6 \%, 7 / 52)$ and was significantly associated with in hospital mortality $(p=0.03)$. According to the WHO 2017 report, tuberculosis remains the most common presentation in HIV-infected individuals and is the leading cause of death among HIV-infected people worldwide [13]. It is noteworthy that an even higher proportion of deaths were accounted for by pneumonia $(19.2 \%, 10 / 52)$ in this study. Given the predominance of atypical radiographic findings in HIV-tuberculosis coinfection $[14,15]$, it is possible that there may have been some overlap between the two conditions, which may have resulted in underestimation of true number of tuberculosis cases.

Anemia is a major hallmark of HIV disease progression and was associated with $48 \%$ (83/173) of admissions and $23 \%(12 / 52)$ of deaths. The pathogenesis of HIVassociated anemia has been well described, with a broad differential including direct myelosuppression by HIV itself leading to impaired/reduced erythropoiesis or increased hemolysis, infiltrative neoplasms, tuberculosis, malaria coinfection in endemic regions and ART/other drug-associated toxicities-most notably zidovudine use $[16,17]$. Of note, about $16 \%(10 / 64)$ of our study participants receiving ART were on a zidovudine-containing regimen. Severe anemia was found to be an independent predictor of early mortality in HIV infection in multiple studies [3, 18, 19] but was not significantly associated with mortality in this study.

The immunological profile of our study participants as determined by the CD4 count distribution and WHO clinical staging highlight the growing problem of latestage HIV presentation felt most keenly in sub-Saharan African countries $[8,20-23]$. In the study participants for whom CD4 count was obtained, 83\% (93/112) met 
Table 4 Factors associated with HIV-related in-hospital mortality

\begin{tabular}{lll}
\hline Characteristics & \multicolumn{2}{l}{ Survival status } \\
\cline { 2 - 3 } & Died & Discharged alive \\
& $N=52(100 \%)$ & $N=121(100 \%)$
\end{tabular}

\begin{tabular}{|c|c|c|c|}
\hline \multicolumn{4}{|l|}{ Age, years } \\
\hline Median (IQR) & $37(29-45)$ & $33(28-42)$ & 0.47 \\
\hline \multicolumn{4}{|l|}{ Gender, N\% } \\
\hline Male & $20(38.5)$ & $50(41.3)$ & \multirow[t]{2}{*}{0.73} \\
\hline Female & $32(61.5)$ & $71(58.7)$ & \\
\hline \multicolumn{4}{|l|}{ Risk factors, N\% } \\
\hline Smoking & $15(28.8)$ & $20(16.5)$ & 0.06 \\
\hline Alcohol use & 18 (34.6) & $21(17.4)$ & 0.01 \\
\hline Illicit drug use & $5(13.5)$ & $4(3.3)$ & 0.09 \\
\hline \multicolumn{4}{|l|}{ CD4 count, cells/ML } \\
\hline Median (IQR) & $70(23-263)$ & $101(29-278)$ & 0.61 \\
\hline Less than $50, \mathrm{~N} \%$ & $12(23.1)$ & $23(19.0)$ & 0.82 \\
\hline \multicolumn{4}{|l|}{ WHO Clinical Staging, N\% } \\
\hline 1 or 2 & $6(11.5)$ & $22(18.2)$ & \multirow[t]{2}{*}{0.28} \\
\hline 3 or 4 & $46(88.5)$ & $99(81.8)$ & \\
\hline \multicolumn{4}{|c|}{ Presenting signs or symptoms, N\% } \\
\hline Fever & $42(80.8)$ & $92(76.0)$ & 0.49 \\
\hline Jaundice & $5(9.6)$ & $2(1.7)$ & 0.02 \\
\hline Weight loss & $30(57.7)$ & $63(52.1)$ & 0.50 \\
\hline Headache & $11(21.2)$ & $22(18.2)$ & 0.65 \\
\hline Seizure & - & $2(1.7)$ & 0.35 \\
\hline Limb weakness & $5(9.6)$ & $3(2.5)$ & 0.04 \\
\hline Loss of consciousness & $5(9.6)$ & $3(2.5)$ & 0.04 \\
\hline \multicolumn{4}{|l|}{ Admitting diagnoses, N\% } \\
\hline Anemia & $25(48.1)$ & $58(47.9)$ & 0.99 \\
\hline Tuberculosis & $7(13.5)$ & $34(28.1)$ & 0.04 \\
\hline Pneumonia & $10(19.2)$ & $20(16.5)$ & 0.67 \\
\hline Diarrheal illness & $8(15.4)$ & $18(14.9)$ & 0.93 \\
\hline Cerebral toxoplasmosis & $6(11.5)$ & $3(2.5)$ & 0.01 \\
\hline Cryptococcal meningitis & $1(1.9)$ & $2(1.7)$ & 0.90 \\
\hline \multicolumn{4}{|l|}{ New Diagnosis, N\% } \\
\hline Yes & $29(55.8)$ & $60(49.6)$ & \multirow[t]{2}{*}{0.46} \\
\hline No & $23(44.2)$ & $61(50.4)$ & \\
\hline \multicolumn{4}{|l|}{ ART status, N\% } \\
\hline Yes & $16(30.8)$ & $48(39.7)$ & \multirow[t]{2}{*}{0.19} \\
\hline No & $36(69.2)$ & $73(60.3)$ & \\
\hline \multicolumn{4}{|l|}{ Length of hospital stay, days } \\
\hline Median & $11(8-16)$ & $12(9-18)$ & 0.48 \\
\hline
\end{tabular}

$I Q R$ interquartile range

the criteria for late-stage HIV disease (i.e., CD4 $<350$ cells $/ \mu \mathrm{L})$, coinciding with the $83.2 \%(144 / 173)$ of patients that were also clinically classified as either WHO stage 3 or 4. Although this suggests that WHO staging could be a reasonably accurate and cost-effective means of classifying HIV disease severity that can be applied in rural areas in Sierra Leone where CD4 cell count testing capability may not be readily, a dedicated study assessing the specificity and sensitivity of this method is warranted. Furthermore, nearly 70\% (78/112) met the criteria for AIDS (i.e., CD $4<200$ cells $/ \mu \mathrm{L}$ ) and over $50 \%$ (56/112) were severely immunosuppressed (i.e., CD4< 100 cells $/ \mu \mathrm{L})$. Recent estimates from elsewhere in subSaharan Africa have also reported a high prevalence of advanced HIV disease among hospitalized HIV patients-83.7 and 97.3\%, in Kenya and the Democratic Republic of Congo, respectively [24]. Late stage HIV diagnosis has been associated with higher morbidity and mortality $[25,26]$ and escalated treatment costs in an already overburdened healthcare system [27]. In our study however, mortality was not associated with latestage HIV presentation, likely due to the sample size of the study.

Other factors significantly associated with in-hospitality were, most prominently, the presence of neurological symptoms-i.e., loss of consciousness $(p=0.04)$ and focal limb weakness $(p=0.04)$, cerebral toxoplasmosis $(p=0.01)$, alcohol use $(p=0.01)$ and jaundice $(p=0.02)$; however, only jaundice emerged as an independent predictor of in-hospital mortality (AOR $0.11,95 \%$ CI [0.020.65 ]; $p=0.01$ ) (Table 5). The etiologies of jaundice in HIV disease span a broad spectrum of conditions. In hyper-endemic countries such as Sierra Leone, jaundice is most often associated with malaria or acute HBV infection, but may also be a prominent clinical feature of alcoholic liver disease, drug-induced hepatotoxicity, tuberculosis-associated granulomatous hepatitis, AIDS cholangiopathy, hepatocellular carcinoma, schistosomiasis, hemangioma, and hepatic adenoma [28, 29]. Several observational studies have shown that HIV coinfection with HBV can lead to rapid more progression to AIDS [30,31], adverse complications such as the immune reconstitution syndrome on ART initiation [32,33], and faster onset of cirrhotic liver disease and hepatocellular carcinoma [33, 34]. Malaria or HBV infection was present in less than $10 \%(16 / 173)$ of our study participants; however, screening was only undertaken in select patients who exhibited suggestive signs or symptoms of liver disease; it is therefore conceivable that the true prevalence of asymptomatic malaria and viral hepatitis may have been much higher in our cohort. Of note, we recently observed a high prevalence of serological markers of chronic HBV infection, i.e., HBsAg $(22 \%, 38 / 175)$ and prior exposure, i.e., total anti-HBc $(83 \%, 175 / 211)$ in a separate cohort of 211 HIV-infected outpatients at Connaught Hospital [35]. In another cohort of HIV infected patients, we also recorded a high prevalence of pretreatment HIV drug resistance (14.2\%) to nucleoside/nucleotide reverse transcriptase inhibitors 
Table 5 Predictors of in-hospital HIV-related mortality

\begin{tabular}{|c|c|c|c|c|}
\hline \multirow[t]{2}{*}{ Characteristics } & \multicolumn{2}{|l|}{ Univariate } & \multicolumn{2}{|l|}{ Multivariate } \\
\hline & OR $(95 \% \mathrm{Cl})$ & $P$ value & AOR (95\% Cl) & $P$ value \\
\hline Middle/older age ( $\geq 45$ years) & $0.97(0.44-2.16)$ & 0.94 & $0.87(0.36-2.10)$ & 0.76 \\
\hline Gender & $0.89(0.46-1.73)$ & 0.73 & $1.48(0.67-3.27)$ & 0.33 \\
\hline Smoking & $2.05(0.95-4.41)$ & 0.06 & $0.77(0.25-2.37)$ & 0.65 \\
\hline Alcohol use & $2.52(1.20-5.28)$ & 0.01 & $0.36(0.11-1.12)$ & 0.08 \\
\hline Illicit drug use & $3.11(0.80-12.10)$ & 0.09 & $0.44(0.09-2.26)$ & 0.33 \\
\hline Jaundice & $6.33(1.19-33.77)$ & 0.02 & $0.11(0.02-0.62)$ & 0.01 \\
\hline Limb Weakness & $4.18(0.96-18.21)$ & 0.04 & $0.47(0.08-2.78)$ & 0.40 \\
\hline Loss of consciousness & $4.18(0.96-18.21)$ & 0.04 & $0.40(0.08-2.12)$ & 0.28 \\
\hline Cerebral toxoplasmosis & $5.13(1.23-21.38)$ & 0.01 & $0.29(0.05-1.48)$ & 0.13 \\
\hline Tuberculosis & $0.40(0.16-0.97)$ & 0.03 & $2.48(0.91-6.70)$ & 0.07 \\
\hline ART status & $0.63(0.32-1.26)$ & 0.19 & $1.28(0.60-2.76)$ & 0.52 \\
\hline
\end{tabular}

$O R$ odds ratio, $A O R$ adjusted odds ratio, $C l$ confidence interval

(NRTIs) among ART-naïve patients $(n=64)$; among the ART-exposed patients $(n=151)$, the prevalence acquired HIV drug resistance to NRTIs was 6-fold higher at $85.2 \%$ [36]. The high levels HIV/HBV co-infection and high cross-resistance rates to NRTIs which are integral treatment regimens for both infections may have far-reaching implications for disease outcomes in HBV hyper-endemic countries such as Sierra Leone and therefore warrant further investigation.

Our study had several limitations, including the small sample size, potentially making the findings not readily generalizable. There was potential selection bias in this study, as patients at the national referral health center would be expected to more severely ill and therefore possibly at higher risk of mortality. Determining admission diagnoses and causes of death were major challenges, given that advanced diagnostic tools which are not readily available in resource-limited settings are needed to determine the etiology of certain infections and AIDS-defining conditions and thus, most diagnoses were presumptive. Incompleteness of the CD4 cell count data was another limitation; this is because the study relied on the availability of point-of-care testing data per standard of care. Furthermore, the study did not track patients in non-medical wards, which may have underestimated the true prevalence of HIV-related admissions. Finally, it was sometimes difficult to ascertain whether patients had previously received medical care elsewhere before presenting to our hospital; additionally, patients who were discharged were not followed up to track their short-term (<30 day) and long term ( $\geq 30$ day) clinical outcomes, which may have skewed some of our observations. Nonetheless, we believe that our study is significant because it offers insight into the morbidity and mortality patterns of hospitalized HIV-infected patients at the main referral tertiary healthcare facility in Sierra
Leone and further provides on update on this important public health topic in the West Africa region.

\section{Conclusions}

In the era of widespread ART availability globally, a high burden of HIV-related admissions and in-hospital mortality were observed at Connaught Hospital in Freetown, the main referral hospital in Sierra Leone. These findings necessitate the implementation of specific measures to enhance early HIV diagnosis and expand treatment access to all HIV-infected patients in Sierra Leone.

\section{Abbreviations}

AFB: Acid fast bacilli; AIDS: Acquired immune deficiency syndrome; Anti$\mathrm{HBC}$ : Total hepatitis B core antibody; AOR: Adjusted odds ratio;

ART: Antiretroviral therapy; Cl: Confidence interval; HBsAg: Hepatitis B surface antigen; HBV: Hepatitis B virus; HCTS: HIV Counselling and Testing Services; HIV: Human immunodeficiency virus; leDEA: International epidemiological Database to Evaluate AIDS; IQR: Interquartile range; OR: Odds ratio; PMTCT: Prevention of Mother to Child Transmission; qSOFA: Quick sequential organ failure assessment score; UNAIDS: United Nations Joint Programme on HIV and AIDS; WHO: World Health Organization

\section{Acknowledgements}

We acknowledge the help and support of the nursing staff on the medical wards at Connaught Hospital, Freetown.

\section{Authors' contributions}

SL, DFJ, JEK and GAY conceptualized the study. SL, DFJ and JEK collected the data. SL, EP, AS-B, FS, RAS and GAY analyzed and interpreted the data. SL, EP, $F S, M S, T S, G F D, W G, R A S$, and GAY drafted the manuscript. All authors read and approved of the final version of the manuscript.

\section{Funding}

This work was not supported by any grants during the enrolment and data collection stages of the study. However, all data analysis, manuscript preparation, and publication costs were funded in part from the Roe Green Travel Medicine Award 2019, University Hospitals Cleveland Medical Center (GAY).

Availability of data and materials

All data and material generated or analyzed during this study are included in this manuscript. Raw de-identified study data are available from the corresponding author upon reasonable request. 


\section{Ethics approval and consent to participate}

Ethical approval was obtained from the Sierra Leone Ethics Scientific and Research Committee for the study. Informed written consent from participants aged $\geq 18$ years and written parental consent was obtained for patients aged 15-18years prior to enrollment in the study.

\section{Consent for publication}

Not applicable, as our manuscript does not contain individual patients' personal information.

\section{Competing interests}

The authors declare that they have no competing interests.

\section{Author details}

${ }^{1}$ College of Medicine and Allied Health Sciences, University of Sierra Leone, Freetown, Sierra Leone. ${ }^{2}$ Department of Medicine, University of Sierra Leone Teaching Hospitals Complex, Freetown, Sierra Leone. ${ }^{3}$ Group of Virology and Pathogenesis, Galicia Sur Health Research Institute (IIS Galicia Sur)-Complexo Hospitalario Universitario de Vigo, SERGAS-UVigo, Vigo, Spain. ${ }^{4}$ Methodology and Statistics Unit, Galicia Sur Health Research Institute (IIS Galicia Sur)-Complexo Hospitalario Universitario de Vigo, SERGAS-UVigo, Vigo, Spain. ${ }^{5}$ National HIV/AIDS Secretariat, Freetown, Sierra Leone. ${ }^{6}$ National AIDS Control Programme, Ministry of Health and Sanitation, Freetown, Sierra Leone. ${ }^{7}$ Department of Medicine, Case Western Reserve University School of Medicine, Cleveland, OH, USA. ${ }^{8}$ Division of Infectious Diseases and HIV Medicine, University Hospitals Cleveland Medical Center, Cleveland, OH, USA. ${ }^{9}$ Johns Hopkins Bloomberg School of Public Health, Baltimore, MD, USA.

\section{Received: 18 January 2019 Accepted: 12 September 2019} Published online: 21 October 2019

\section{References}

1. UNAIDS 2018. Global HIV \& AIDS statistics - 2018 fact sheet. http://www. unaids.org/sites/default/files/media_asset/UNAIDS_FactSheet_en.pdf.

2. UNAIDS 2018. Region: west and Central Africa. http://www.unaids.org/en/ regionscountries/westandcentralafrica.

3. Obiako RO, Muktar HM, Adeiza M, et al. Morbidity and mortality patterns of hospitalised adult HIV/AIDS patients in the era of highly active antiretroviral therapy: a 4-year retrospective review from Zaria, northern Nigeria. AIDS Res Treat. 2012;2012:940580.

4. Saavedra A, Campinha-Bacote N, Hajjar M, et al. Causes of death and factors associated with early mortality of HIV-infected adults admitted to Korle-Bu teaching hospital. Pan Afr Med J. 2017;27:48

5. Lewden C, Drabo YJ, Zannou DM, et al. Disease patterns and causes of death of hospitalized HIV-positive adults in West Africa: a multicountry survey in the antiretroviral treatment era. J Int AIDS Soc. 2014;17:1-12.

6. Yendewa GA, Poveda E, Yendewa SA, et al. HIV/AIDS in Sierra Leone: characterizing the hidden epidemic. AIDS Rev. 2018;20:105-14.

7. UNAIDS 2016. Country Sierra Leone 2016. http://www.unaids.org/en/ regionscountries/countries/sierraleone.

8. Yendewa GA, Poveda E, Lakoh S, et al. High prevalence of late-stage diagnosis in newly diagnosed HIV patients in Sierra Leone. Open Forum Infect Dis. 2018;5:ofy208.

9. Faye B, Mbow M, Cheikh Seck M, et al. Evaluation of PIMATM CD4 system for decentralization of immunological monitoring of HIV-infected patients in Senegal. PLoS One. 2016;11:e0154000

10. Angus DC, Seymour CW, Coopersmith CM, et al. A framework for the development and interpretation of different sepsis. Definitions and clinical criteria. Crit Care Med. 2016;44:e113-21.

11. WHO. Consolidated guidelines on the use of antiretroviral drugs for treating and preventing HIV infection: recommendations for a public health approach. 2nd ed; 2016. http://apps.who.int/iris/bitstream/handle/10665/2 08825/9789241549684_eng.pdf?sequence=1

12. Statistics Sierra Leone. Sierra Leone Health and Demographic Survey 2013. https://dhsprogram.com/pubs/pdf/fr297/fr297.pdf.

13. WHO 2018. HIV-associated tuberculosis. https://www.who.int/tb/areas-ofwork/tb-hiv/tbhiv_factsheet.pdf?ua=1.

14. Long $R$, Maycher $\bar{B}$, Scalcini $M$, et al. The chest roentgenogram in pulmonary tuberculosis patients seropositive for human immunodeficiency virus type 1. Chest. 1991;99:123.
15. Barnes PF, Bloch AB, Davidson PT, et al. Tuberculosis in patients with human immunodeficiency virus infection. N Engl J Med. 1991;324:1644.

16. Volberding PA, Levine AM, Dieterich $D$, et al. Anemia in HIV working group. Anemia in HIV infection: clinical impact and evidence-based management strategies. Clin Infect Dis. 2004;38:1454-63.

17. Naing C, Sandhu NK, Wai VN. The effect of malaria and HIV co-infection on anemia: a meta-analysis. Medicine (Baltimore). 2016;95:e3205.

18. O'Brien ME, Kupka R, Msamanga Gl, et al. Anemia is an independent predictor of mortality and immunologic progression of disease among women with HIV in Tanzania. J Acquir Immune Defic Syndr. 2005;40:219.

19. Mocroft A, Kirk O, Barton SE, et al. Anaemia is an independent predictive marker for clinical prognosis in HIV-infected patients from across Europe. EuroSIDA study group. Anaemia is an independent predictive marker for clinical prognosis in HIVinfected patients from across Europe. AIDS. 1999;13:943-50.

20. Kujawski SA, Lamb MR, Lahuerta M, et al. Advanced human immunodeficiency virus disease at diagnosis in Mozambique and Swaziland. Open Forum Infect Dis. 2017;4:ofx156.

21. Gesesew HA, Ward P, Woldemichael K, Mwanri L. Late presentation for HIV care in Southwest Ethiopia in 2003-2015: prevalence, trend, outcomes and risk factors. BMC Infect Dis. 2018;18:59.

22. Hønge BL, Jespersen S, Aunsborg J, et al. High prevalence and excess mortality of late presenters among HIV-1, HIV-2 and HIV-1/2 dually infected patients in Guinea-Bissau - a cohort study from West Africa. Pan Afr Med J. 2016;25:40.

23. Yendewa GA. Hot news: new insights from leDEA and COHERE on global trends in CD4 counts at ART initiation. AIDS Rev. 2018;20:174-5.

24. Ousley J, Niyibizi AA, Wanjala S, et al. High proportions of patients with advanced HIV are antiretroviral therapy experienced: hospitalization outcomes from 2 sub-Saharan African sites. Clin Infect Dis. 2018;66:S126-32.

25. Grinsztejn B, Hosseinipour MC, Ribaudo HJ, et al. Effects of early versus delayed initiation of antiretroviral treatment on clinical outcomes of HIV-1 infection: results from the phase 3 HPTN 052 randomised controlled trial. Lancet Infect Dis. 2014;14:281-90.

26. Lawn SD, Harries AD, Anglaret X, et al. Early mortality among adults accessing antiretroviral treatment programmes in sub-Saharan Africa. AIDS. 2008;22:1897-908.

27. Fleishman JA, Yehia BR, Moore RD, Gebo KA. The economic burden of late entry into medical care for patients with HIV infection. Med Care. 2010;48: 1071-9.

28. Chalasani N, Wilcox CM. Etiology, evaluation, and outcome of jaundice in patients with acquired immunodeficiency syndrome. Hepatology. 1996;23:728-33.

29. Ocama P, Katwere M, Piloya T, et al. The spectrum of liver diseases in HIV infected individuals at an HIV treatment clinic in Kampala, Uganda. Afr Health Sci. 2008;8:8-12.

30. Eskild A, Magnus P, Petersen G, et al. Hepatitis B antibodies in HIV-infected homosexual men are associated with more rapid progression to AIDS. AIDS. 1992;6:571.

31. Hoffmann CJ, Seaberg EC, Young S, et al. Hepatitis B and long-term HIV outcomes in coinfected HAART recipients. AIDS. 2009;23:1881.

32. Drake A, Mijch A, Sasadeusz J. Immune reconstitution hepatitis in HIV and hepatitis B co infection, despite lamivudine as part of HAART. Clin Infect Dis. 2004;39:129-32 Hoffmann CJ.

33. Hoffmann CJ, Charalambous S, Thio CL, et al. Hepatotoxicity in an African antiretroviral therapy cohort: the effect of tuberculosis and hepatitis B. AIDS. 2007;21:1301-8

34. Thio CL, Seaberg EC, Skolasky R Jr, et al. HIV-1, hepatitis B virus, and risk of liverrelated mortality in the multicenter cohort study. Lancet. 2002;360:1921.

35. Pinato DJ, Allara E, Chen TY, et al. Influence of HIV infection on the natural history of hepatocellular carcinoma: results from a global multicohort study. J Clin Oncol. 2019;37:296-304.

36. Yendewa GA, Sahr F, Aguilera A, et al. Seroprevalence of hepatitis B, hepatitis $C$ and human T-cell lymphotropic virus infections in human immunodeficiency virus-infected patients in Sierra Leone. Am J Trop Med Hyg. 2019;100:1521-4.

37. Yendewa GA, Sahr F, Lakoh S, et al. Prevalence of drug resistance mutations among ART-naïve and experienced HIV-infected patients in Sierra Leone. J Antimicrob Chemother. 2019;74:2024-9.

\section{Publisher's Note}

Springer Nature remains neutral with regard to jurisdictional claims in published maps and institutional affiliations. 